Acta Math. Hungar., 128 (3) (2010), 265-286.

DOI: $10.1007 / \mathrm{s} 10474-010-9189-4$

First published online May 11, 2010

\title{
ON THE SHIFT SEMIGROUP ON THE HARDY SPACE OF DIRICHLET SERIES
}

\author{
A. OLOFSSON \\ Mathematics, Faculty of Science, Centre for Mathematical Sciences, Lund University, \\ P.O. Box 118, SE-221 00 Lund, Sweden \\ e-mail: olofsson@maths.lth.se \\ (Received August 25, 2009; revised January 11, 2010; accepted January 15, 2010)
}

\begin{abstract}
We develop a Wold decomposition for the shift semigroup on the Hardy space $\mathcal{H}^{2}$ of square summable Dirichlet series convergent in the half-plane $\Re(s)>1 / 2$. As an application we have that a shift invariant subspace of $\mathcal{H}^{2}$ is unitarily equivalent to $\mathcal{H}^{2}$ if and only if it has the form $\varphi \mathcal{H}^{2}$ for some $\mathcal{H}^{2}$-inner function $\varphi$.
\end{abstract}

\section{Introduction}

Let $\mathcal{E}$ be an auxiliary Hilbert space and denote by $\mathcal{H}^{2}(\mathcal{E})$ the space of all $\mathcal{E}$-valued Dirichlet series of the form

$$
f(s)=\sum_{n=1}^{\infty} a_{n} n^{-s},
$$

where $a_{n} \in \mathcal{E}$ for $n \geqq 1$, with finite norm

$$
\|f\|_{\mathcal{H}^{2}}^{2}=\sum_{n=1}^{\infty}\left\|a_{n}\right\|^{2}<+\infty .
$$

The Dirichlet series $(0.1)$ of a function $f \in \mathcal{H}^{2}(\mathcal{E})$ converges in the half-plane $\mathbb{C}_{1 / 2}$, where

$$
\mathbb{C}_{\alpha}=\{s=\sigma+i t \in \mathbb{C}: \Re(s)=\sigma>\alpha\}
$$

for $\alpha$ real, as follows by an application of the Cauchy-Schwarz inequality. It is easy to see that the space $\mathcal{H}^{2}(\mathcal{E})$ becomes in $\mathbb{C}_{1 / 2}$ a Hilbert space of $\mathcal{E}$-valued analytic functions.

Key words and phrases: shift semigroup, Hardy space of Dirichlet series, Wold decomposition, invariant subspace.

2000 Mathematics Subject Classification: primary: 47A15, 47B32; secondary: 30B50, 46E22. 
In the scalar context, the study of the space $\mathcal{H}^{2}$ was initiated by Hedenmalm, Lindqvist and Seip [11] as a Dirichlet series analogue of the classical Hardy space $H^{2}(\mathbb{D})$ of square summable power series in the unit disc $\mathbb{D}$. A characteristic feature of the space $\mathcal{H}^{2}$ is that of its reproducing kernel function being essentially the Riemann zeta function from number theory: $K_{\mathcal{H}^{2}}\left(s, s^{\prime}\right)=\zeta\left(s+\overline{s^{\prime}}\right)$ for $s, s^{\prime} \in \mathbb{C}_{1 / 2}$, where $\zeta(s)=\sum_{n \geqq 1} 1 / n^{s}$.

For every positive integer $n \in \mathbb{Z}^{+}$we have a natural operator $S(n)$ acting on $\mathcal{H}^{2}$ defined by

$$
S(n) f(s)=n^{-s} f(s), \quad s \in \mathbb{C}_{1 / 2},
$$

for functions $f \in \mathcal{H}^{2}$. This provides us with a function $S: \mathbb{Z}^{+} \ni n \mapsto S(n)$ which is easily seen to be a multiplicative semigroup of operators in the sense that

$$
S(n m)=S(n) S(m), \quad n, m \in \mathbb{Z}^{+},
$$

and $S(1)=I$, where $I$ is the identity operator. We call this semigroup $S$ the shift semigroup on $\mathcal{H}^{2}$. One can think of the shift semigroup as a counterpart for the space $\mathcal{H}^{2}$ of the classical shift operator on the Hardy space $H^{2}(\mathbb{D})$ given by multiplication by the complex coordinate.

Some properties of the shift semigroup $S$ are more or less evident: For every integer $n \geqq 2$ the operator $S(n)$ is a pure isometry. For relatively prime integers $n, m \in \mathbb{Z}^{+}$the operators $S(n)$ and $S(m)^{*}$ commute. It is also fairly straightforward to see that

$$
\bigcap_{n=1}^{\infty}\left(\bigvee_{k=n}^{\infty} S\left(p_{k}\right)\left(\mathcal{H}^{2}\right)\right)=\{0\},
$$

where $p_{1}=2, p_{2}=3, p_{3}=5, \ldots$ is the sequence of prime numbers (see Proposition 1.1). Recall that the symbol $\bigvee$ is used to denote a closed linear span.

The main purpose of the present paper is to prove that the three properties quoted in the previous paragraph completely determine the shift semigroup $S$ up to unitary equivalence allowing for a general multiplicity $\mathcal{E}$ as indicated above. The unitary equivalence can be chosen of a certain canonical form similar to what has previously been done for pure isometries and some related classes of operators using a so-called Wold decomposition (see for instance $[2,9,16,21])$. Let us proceed to describe our results.

Let $\mathcal{H}$ be a Hilbert space and denote by $\mathcal{L}(\mathcal{H})$ the space of all bounded linear operators on $\mathcal{H}$. Let $T: \mathbb{Z}^{+} \rightarrow \mathcal{L}(\mathcal{H})$ be a multiplicative semigroup of operators satisfying the analogous properties to those quoted above for 
the shift semigroup; the exact properties are listed as conditions (1)-(3) in Section 2. We call the subspace

$$
\mathcal{E}=\mathcal{H} \ominus\left(\bigvee_{k=1}^{\infty} T\left(p_{k}\right)(\mathcal{H})\right)
$$

of $\mathcal{H}$ for the wandering subspace for $T$, and we denote by $P$ the orthogonal projection of $\mathcal{H}$ onto $\mathcal{E}$. We associate to every element $x \in \mathcal{H}$ the $\mathcal{E}$-valued function given by the Dirichlet series

$$
(V x)(s)=\sum_{n=1}^{\infty}\left(P T(n)^{*} x\right) n^{-s}
$$

which is initially seen to converge in the smaller half-plane $\mathbb{C}_{1}$. The task at hand is then to prove that the map $V: x \mapsto V x$ gives an isometry of $\mathcal{H}$ onto $\mathcal{H}^{2}(\mathcal{E})$ which intertwines $T$ with the shift semigroup $S$ in the sense that

$$
V T(n)=S(n) V, \quad n \in \mathbb{Z}^{+} .
$$

The map $V$ then provides a unitary equivalence of semigroups $T$ and $S$. The details of this program are carried out in Sections 2-3 in the paper. In Section 1 we review the Wold decomposition of an isometry and show that the shift semigroup has the properties quoted above.

It should be mentioned here that Wold decompositions for pairs of doubly commuting isometries have been studied by Słociński [22]. We recall that two operators $T_{1}, T_{2} \in \mathcal{L}(\mathcal{H})$ are said to doubly commute if $T_{1} T_{2}=T_{2} T_{1}$ and $T_{1} T_{2}^{*}=T_{2}^{*} T_{1}$. Popovici [18] has studied Wold decompositions for pairs of commuting isometries dropping the assumption of double commutativity.

Following Bohr [6] we can for the $k$-th prime $p_{k}$ identify $p_{k}^{-s}$ with a complex variable $z_{k}(k=1,2, \ldots)$ to make the Dirichlet series $(0.1)$ correspond to a power series

$$
f(z)=\sum_{n=1}^{\infty} a_{n} z_{1}^{\nu_{1}} z_{2}^{\nu_{2}} \ldots z_{m}^{\nu_{m}}
$$

in infinitely many variables $z_{1}, z_{2}, \ldots$, where in the sum $n=p_{1}^{\nu_{1}} p_{2}^{\nu_{2}} \ldots p_{m}^{\nu_{m}}$ is the factorization of $n$ into a product of prime powers. The power series obtained from functions $f \in \mathcal{H}^{2}$ in this way extend by means of bounded point evaluations to the set

$$
\Delta=\left\{\left\{z_{k}\right\}_{k=1}^{\infty}:\left|z_{k}\right|<1 \text { for } k \geqq 1 \text { and } \sum_{k=1}^{\infty}\left|z_{k}\right|^{2}<+\infty\right\}
$$


(see Cole and Gamelin [7] for details). It is natural to think of $\mathcal{H}^{2}$ as an equivalent of the Hardy space $H^{2}(\Delta)$ on the set $\Delta=\mathbb{D}^{\omega} \cap \ell^{2}$. The operator $S\left(p_{k}\right)$ on $\mathcal{H}^{2}$ corresponds to the shift $S_{k}$ on $H^{2}(\Delta)$ which is the operator given by multiplication by the variable $z_{k}(k=1,2, \ldots)$. By the analysis of Sections $2-3$ in this paper we have that an infinite sequence $\left\{T_{k}\right\}_{k=1}^{\infty}$ of operators on a Hilbert space $\mathcal{H}$ is unitarily equivalent to the shift sequence $\left\{S_{k}\right\}_{k=1}^{\infty}$ on a vector-valued Hardy space $H^{2}(\Delta, \mathcal{E})$ if and only if every operator $T_{k}$ is a pure isometry, the operators $T_{j}$ and $T_{k}$ doubly commute for $j \neq k$, and the condition

$$
\bigcap_{n=1}^{\infty}\left(\bigvee_{k=n}^{\infty} T_{k}(\mathcal{H})\right)=\{0\}
$$

is satisfied. This result is in some contrast to the case of a finite operator tuple $\left(T_{1}, \ldots, T_{n}\right) \in \mathcal{L}(\mathcal{H})^{n}$ on a Hilbert space $\mathcal{H}$ which is unitarily equivalent to the shift tuple $\left(S_{1}, \ldots, S_{n}\right)$ on a vector-valued polydisc Hardy space $H^{2}\left(\mathbb{D}^{n}, \mathcal{E}\right)$ if and only if every operator $T_{k}$ is a pure isometry and the operators $T_{j}$ and $T_{k}$ doubly commute for all $j \neq k$ (see [1,22]).

It should be pointed out that condition (0.5) does not follow from the mere fact that the operator sequence $\left\{T_{k}\right\}_{k=1}^{\infty}$ is such that every operator $T_{k}$ is a pure isometry and the operators $T_{j}$ and $T_{k}$ doubly commute for $j \neq k$. A multiplicative semigroup of operators $T: \mathbb{Z}^{+} \rightarrow \mathcal{L}(\mathcal{H})$ satisfying conditions (1)-(2) in Section 2 can have null wandering subspace in the sense of (0.4). We give an example of this phenomenon in Section 4.

By a shift invariant subspace of $\mathcal{H}^{2}$ we mean a closed subspace $\mathcal{I}$ of $\mathcal{H}^{2}$ such that $S(n)(\mathcal{I}) \subset \mathcal{I}$ for every positive integer $n \in \mathbb{Z}^{+}$. As an application of the Wold decomposition discussed in Sections 2-3 we give in Section 5 some operator-theoretic characteristics of the shift invariant subspaces $\mathcal{I}$ of $\mathcal{H}^{2}$ that have the form $\mathcal{I}=\varphi \mathcal{H}^{2}$ for some $\mathcal{H}^{2}$-inner function $\varphi$ (see Theorem 5.1). By an $\mathcal{H}^{2}$-inner function we mean a function $\varphi$ in $\mathcal{H}^{2}$ of unit norm such that $S(n) \varphi \perp S(m) \varphi$ in $\mathcal{H}^{2}$ for $n, m \in \mathbb{Z}^{+}$with $n \neq m$. It is fairly straightforward to see that an $\mathcal{H}^{2}$-inner function is the same thing as an isometric multiplier on $\mathcal{H}^{2}$. By a result of Hedenmalm, Lindqvist and Seip [11, Theorem 3.1] a bounded multiplier $m$ on $\mathcal{H}^{2}$ corresponds to a germ of a convergent Dirichlet series

$$
m(s)=\sum_{n=1}^{\infty} m_{n} n^{-s}
$$

that extends analytically to a bounded analytic function in the right halfplane $\mathbb{C}_{0}$ with operator norm given by $\|m\|=\sup _{s \in \mathbb{C}_{0}}|m(s)|$. The space of such Dirichlet series $m$ is denoted $\mathcal{H}^{\infty}$. In a recent paper Saksman and 
Seip [20, Theorem 2] have shown that functions in $\mathcal{H}^{\infty}$ in a natural sense have boundary values a.e. on the character group $\Xi$. In this context an $\mathcal{H}^{2}$ inner function $\varphi$ is a function $\varphi$ in $\mathcal{H}^{\infty}$ such that $|\varphi(\chi)|=1$ for a.e. $\chi \in \Xi$ with respect to Haar measure.

The author thanks the referee for a careful reading of the manuscript.

\section{Preliminaries}

Let us recall first the Wold decomposition of an isometry. A Hilbert space operator $T \in \mathcal{L}(\mathcal{H})$ is called an isometry if $T^{*} T=I$ in $\mathcal{L}(\mathcal{H})$, where $I$ is the identity operator. An isometry $T \in \mathcal{L}(\mathcal{H})$ is called pure if

$$
\bigcap_{k=1}^{\infty} T^{k}(\mathcal{H})=\{0\}
$$

The subspace $\mathcal{E}=\mathcal{H} \ominus T(\mathcal{H})$ is called the wandering subspace for $T \in \mathcal{L}(\mathcal{H})$.

Lemma 1.1. Let $T \in \mathcal{L}(\mathcal{H})$ be a pure isometry. Then

$$
\mathcal{H}=\bigvee_{k=0}^{\infty} T^{k}(\mathcal{E})
$$

where $\mathcal{E}=\mathcal{H} \ominus T(\mathcal{H})$ is the wandering subspace for $T$.

Proof. See Halmos [9] or Sz.-Nagy and Foias [23, Section I.1].

Recall that the symbol $\bigvee$ denotes a closed linear span. Since in Lemma 1.1 the ranges $T^{k}(\mathcal{E})(k \geqq 0)$ are mutually orthogonal it is evident that the space $\mathcal{H}$ decomposes as an orthogonal sum

$$
\mathcal{H}=\bigoplus_{k=0}^{\infty} T^{k}(\mathcal{E})
$$

where $\mathcal{E}$ is the wandering subspace for $T$.

Let us record also some formulas for the shifts acting on functions in $\mathcal{H}^{2}$. The operator $S(n)$ acts as

$$
S(n) f(s)=n^{-s} f(s)=\sum_{k=1}^{\infty} \hat{f}(k)(n k)^{-s}=\sum_{k: n \mid k} \hat{f}(k / n) k^{-s}, \quad s \in \mathbb{C}_{1 / 2}
$$

where

$$
f(s)=\sum_{k=1}^{\infty} \hat{f}(k) k^{-s}, \quad s \in \mathbb{C}_{1 / 2}
$$


is the Dirichlet series expansion of $f \in \mathcal{H}^{2}$. A similar calculation shows that the adjoint shift $S(n)^{*}$ acts as

$$
S(n)^{*} f(s)=\sum_{k=1}^{\infty} \hat{f}(n k) k^{-s}, \quad s \in \mathbb{C}_{1 / 2},
$$

on functions $f \in \mathcal{H}^{2}$ given by (1.2).

The next proposition gives some basic properties of the shift $S$.

Proposition 1.1. For every integer $n \geqq 2$ the operator $S(n) \in \mathcal{L}\left(\mathcal{H}^{2}\right)$ is a pure isometry. For relatively prime integers $n, m \in \mathbb{Z}^{+}$the operators $S(n)$ and $S(m)^{*}$ commute: $S(n) S(m)^{*}=S(m)^{*} S(n)$. Furthermore

$$
\bigcap_{n=1}^{\infty}\left(\bigvee_{k=n}^{\infty} S\left(p_{k}\right)\left(\mathcal{H}^{2}\right)\right)=\{0\}
$$

where $\left\{p_{k}\right\}_{k=1}^{\infty}$ is the sequence of prime numbers.

Proof. A calculation using formulas (1.1) and (1.3) above gives that $S(n)^{*} S(n) f=f$ for $f \in \mathcal{H}^{2}$ showing that $S(n) \in \mathcal{L}\left(\mathcal{H}^{2}\right)$ is an isometry. In a similar manner it is straightforward to verify that

$$
S(n) S(m)^{*} f=S(m)^{*} S(n) f
$$

for $f \in \mathcal{H}^{2}$ if $n, m \in \mathbb{Z}^{+}$are relatively prime.

As a consequence of (1.1) the range $S(n)\left(\mathcal{H}^{2}\right)$ consists of all functions $f \in \mathcal{H}^{2}$ such that $\hat{f}(k)=0$ for all positive integers $k \in \mathbb{Z}^{+}$not divisible by $n$, or put another way that

$$
S(n)\left(\mathcal{H}^{2}\right)=\left\{f \in \mathcal{H}^{2}: \operatorname{supp}(\hat{f}) \subset n \mathbb{Z}^{+}\right\},
$$

where $\operatorname{supp}(\hat{f})$ denotes the support of $\hat{f}$ as a function on $\mathbb{Z}^{+}$. An application of (1.4) gives that the isometry $S(n)$ is pure for $n \geqq 2$. An iteration of (1.4) gives that

$$
\bigvee_{k=n}^{\infty} S\left(p_{k}\right)\left(\mathcal{H}^{2}\right)=\left\{f \in \mathcal{H}^{2}: \operatorname{supp}(\hat{f}) \subset \bigcup_{k=n}^{\infty}\left(p_{k} \mathbb{Z}^{+}\right)\right\} .
$$

Next taking an intersection we find that

$$
\bigcap_{n=1}^{\infty}\left(\bigvee_{k=n}^{\infty} S\left(p_{k}\right)\left(\mathcal{H}^{2}\right)\right)=\left\{f \in \mathcal{H}^{2}: \operatorname{supp}(\hat{f}) \subset \bigcap_{n=1}^{\infty}\left(\bigcup_{k=n}^{\infty}\left(p_{k} \mathbb{Z}^{+}\right)\right)\right\}=\{0\},
$$

where the last equality is obvious since $\bigcap_{n \geqq 1} \bigcup_{k \geqq n}\left(p_{k} \mathbb{Z}^{+}\right)=\emptyset$. 


\section{Construction of the Wold map $V$}

Let $\mathcal{H}$ be a Hilbert space and denote by $\mathcal{L}(\mathcal{H})$ the space of all bounded linear operators on $\mathcal{H}$. We shall first consider a multiplicative semigroup $T: \mathbb{Z}^{+} \rightarrow \mathcal{L}(\mathcal{H})$ of operators satisfying the conditions:

(1) The operator $T(p) \in \mathcal{L}(\mathcal{H})$ is a pure isometry for every prime number $p$.

(2) For different primes $p$ and $q$, the operators $T(p)$ and $T(q)$ doubly commute.

Let $T: \mathbb{Z}^{+} \rightarrow \mathcal{L}(\mathcal{H})$ be a multiplicative semigroup of operators satisfying (1)-(2). We shall make use of the subspace

$$
\mathcal{E}=\mathcal{H} \ominus\left(\bigvee_{k=1}^{\infty} T\left(p_{k}\right)(\mathcal{H})\right)=\bigcap_{k=1}^{\infty} \operatorname{ker} T\left(p_{k}\right)^{*}
$$

of $\mathcal{H}$, where $\left\{p_{k}\right\}_{k=1}^{\infty}$ is the sequence of prime numbers. This subspace $\mathcal{E}$ has the property that

$$
T(n)(\mathcal{E}) \perp T(m)(\mathcal{E}) \quad \text { in } \quad \mathcal{H}
$$

for all $n, m \in \mathbb{Z}^{+}$with $n \neq m$. We call a subspace $\mathcal{E}$ of $\mathcal{H}$ with the property that $T(n)(\mathcal{E}) \perp T(m)(\mathcal{E})$ in $\mathcal{H}$ for all relatively prime integers $n, m \in \mathbb{Z}^{+}$ with $n \neq m$ for a wandering subspace for the semigroup $T$. The particular wandering subspace $\mathcal{E}$ in (2.1) is referred to as the wandering subspace for $T: \mathbb{Z}^{+} \rightarrow \mathcal{L}(\mathcal{H})$. The following theorem illustrates the importance of this notion of wandering subspace.

Theorem 2.1. Let $T: \mathbb{Z}^{+} \rightarrow \mathcal{L}(\mathcal{H})$ be a multiplicative semigroup satisfying conditions (1)-(2), and denote by $\mathcal{E}$ the wandering subspace for $T$ given by (2.1). Then

$$
\bigvee_{n=1}^{\infty} T(n)(\mathcal{E})=\mathcal{H} \ominus\left(\bigcap_{n=1}^{\infty}\left(\bigvee_{k=n}^{\infty} T\left(p_{k}\right)(\mathcal{H})\right)\right)
$$

Proof. We shall iterate the result of Lemma 1.1. Consider the subspaces

$$
\mathcal{H}_{n}=\mathcal{H} \ominus\left(\bigvee_{k \geqq n} T\left(p_{k}\right)(\mathcal{H})\right), \quad n=1,2,3, \ldots,
$$

of $\mathcal{H}$. Notice that $\mathcal{H}_{1}=\mathcal{E}$ is the wandering subspace given by (2.1). Observe also that the subspace $\mathcal{H}_{n+1}$ is reducing for the operator $T\left(p_{n}\right)$ for $n=1,2, \ldots$. 
We shall next prove that

$$
\mathcal{H}_{n+1} \ominus T\left(p_{n}\right)\left(\mathcal{H}_{n+1}\right)=\mathcal{H}_{n}, \quad n=1,2, \ldots
$$

It is obvious that $\mathcal{H}_{n}$ is contained in the space on the left hand side in (2.3). To prove the reverse inclusion it suffices to show that if $x \in \mathcal{H}_{n+1}$ is such that $x \perp T\left(p_{n}\right)\left(\mathcal{H}_{n+1}\right)$, then $x \perp T\left(p_{n}\right)(\mathcal{H})$. For this purpose let $y \in \mathcal{H}$ and write $y=y_{1}+y_{2}$, where $y_{1} \in \mathcal{H}_{n+1}$ and $y_{2} \in \mathcal{H} \ominus \mathcal{H}_{n+1}$. Since the subspace $\mathcal{H}_{n+1}$ reduces $T\left(p_{n}\right)$, we have that $T\left(p_{n}\right) y_{2} \in \mathcal{H} \ominus \mathcal{H}_{n+1}$. Now

$$
\left\langle x, T\left(p_{n}\right) y\right\rangle=\left\langle x, T\left(p_{n}\right) y_{1}\right\rangle+\left\langle x, T\left(p_{n}\right) y_{2}\right\rangle=0,
$$

where $\left\langle x, T\left(p_{n}\right) y_{1}\right\rangle=0$ because $y_{1} \in \mathcal{H}_{n+1}$ and $\left\langle x, T\left(p_{n}\right) y_{2}\right\rangle=0$ because $T\left(p_{n}\right) y_{2} \in \mathcal{H} \ominus \mathcal{H}_{n+1}$.

Notice that the operator $T\left(p_{n}\right)$ restricted to $\mathcal{H}_{n+1}$ is a pure isometry and that the wandering subspace for $\left.T\left(p_{n}\right)\right|_{\mathcal{H}_{n+1}}$ is $\mathcal{H}_{n}$ by $(2.3)$. By Lemma 1.1,

$$
\mathcal{H}_{n+1}=\bigvee_{k \geqq 0} T\left(p_{n}\right)^{k}\left(\mathcal{H}_{n}\right), \quad n=1,2, \ldots
$$

Proceeding inductively we find that

$$
\bigvee_{k_{1}, k_{2}, \ldots, k_{n} \geqq 0} T\left(p_{1}^{k_{1}} p_{2}^{k_{2}} \ldots p_{n}^{k_{n}}\right)(\mathcal{E})=\mathcal{H} \ominus\left(\bigvee_{k=n+1}^{\infty} T\left(p_{k}\right) \mathcal{H}\right)
$$

for positive integers $n$. By this last formula the conclusion of the theorem follows.

We remark that

$$
\bigvee_{n=1}^{\infty} T(n)(\mathcal{E})=\bigoplus_{n=1}^{\infty} T(n)(\mathcal{E})
$$

in Theorem 2.1 by the orthogonality property (2.2). In the following developments we shall rephrase this last equality in a somewhat more appealing form.

Let $T: \mathbb{Z}^{+} \rightarrow \mathcal{L}(\mathcal{H})$ be as in Theorem 2.1. It is straightforward to see that the subspace

$$
\mathcal{H}_{0}=\bigcap_{n=1}^{\infty}\left(\bigvee_{k=n}^{\infty} T\left(p_{k}\right)(\mathcal{H})\right)
$$

of $\mathcal{H}$ is reducing for $T$, that is, the inclusions $T(n)\left(\mathcal{H}_{0}\right) \subset \mathcal{H}_{0}$ and $T(n)^{*}\left(\mathcal{H}_{0}\right)$ $\subset \mathcal{H}_{0}$ hold for every $n \in \mathbb{Z}^{+}$. It should be pointed out that the subspace $\mathcal{H}_{0}$ 
in (2.4) can be nonzero assuming only conditions (1)-(2). We shall construct such an example in Section 4.

Motivated by Theorem 2.1 we are led to consider semigroups $T: \mathbb{Z}^{+} \rightarrow$ $\mathcal{L}(\mathcal{H})$ that in addition to $(1)-(2)$ above also satisfy the condition:

(3) The semigroup $T: \mathbb{Z}^{+} \rightarrow \mathcal{L}(\mathcal{H})$ is such that

$$
\bigcap_{n=1}^{\infty}\left(\bigvee_{k=n}^{\infty} T\left(p_{k}\right)(\mathcal{H})\right)=\{0\}
$$

where $\left\{p_{k}\right\}_{k=1}^{\infty}$ is the sequence of prime numbers.

Remark that condition (3) inherits to invariant subspaces. The condition also has some resemblance to the notion of pureness of an isometry.

We next consider the orthogonal projection onto the wandering subspace.

Lemma 2.1. Let $T: \mathbb{Z}^{+} \rightarrow \mathcal{L}(\mathcal{H})$ be a multiplicative semigroup satisfying conditions (1)-(2). Then the infinite product

$$
P=\prod_{k=1}^{\infty}\left(I-T\left(p_{k}\right) T\left(p_{k}\right)^{*}\right) \quad \text { in } \quad \mathcal{L}(\mathcal{H})
$$

converges in the strong operator topology in $\mathcal{L}(\mathcal{H})$. The operator $P$ is the orthogonal projection of $\mathcal{H}$ onto the wandering subspace $\mathcal{E}$ for $T$ given by (2.1).

Proof. The operator $P_{k}=I-T\left(p_{k}\right) T\left(p_{k}\right)^{*}$ is the orthogonal projection of $\mathcal{H}$ onto the wandering subspace $\mathcal{H} \ominus T\left(p_{k}\right)(\mathcal{H})$ for $T\left(p_{k}\right)$. Notice that the projections $P_{j}$ and $P_{k}$ commute since the operators $T\left(p_{j}\right)$ and $T\left(p_{k}\right)$ doubly commute for $j \neq k$. The finite product $\prod_{k=1}^{N} P_{k}$ is the orthogonal projection of $\mathcal{H}$ onto the subspace

$$
\mathcal{H} \ominus\left(\bigvee_{k=1}^{N} T\left(p_{k}\right)(\mathcal{H})\right)=\bigcap_{k=1}^{N}\left(\mathcal{H} \ominus T\left(p_{k}\right)(\mathcal{H})\right)
$$

of $\mathcal{H}$. Standard Hilbert space considerations give that the operator limit

$$
P=\lim _{N \rightarrow \infty} \prod_{k=1}^{N} P_{k} \quad \text { in } \quad \mathcal{L}(\mathcal{H})
$$

exists in the strong operator topology in $\mathcal{L}(\mathcal{H})$ and equals the orthogonal projection of $\mathcal{H}$ onto $\mathcal{E}$.

For easy reference we record the following lemma. 
Lemma 2.2. Let $T: \mathbb{Z}^{+} \rightarrow \mathcal{L}(\mathcal{H})$ be a multiplicative semigroup satisfying conditions (1)-(2). Then $T(m)^{*} T(n)=T(m / n)^{*}$ if $n$ divides $m$. Also, if $n$ does not divide $m$, then $P T(m)^{*} T(n)=0$, where $P$ is the orthogonal projection of $\mathcal{H}$ onto the wandering subspace $\mathcal{E}$ for $T$.

Proof. First if $n$ divides $m$, then $T(m)^{*} T(n)=T(m / n)^{*} T(n)^{*} T(n)=$ $T(m / n)^{*}$ since $T(n)$ is an isometry.

We next consider the case when $n$ does not divide $m$. Considering the prime factorizations of $n$ and $m$ we have a prime power $p^{k}$ that divides $n$ but not $m$. Taking a smaller exponent $k$ if necessary, we can arrange that also $p^{k-1}$ divides $m$. Now

$$
\begin{aligned}
& T(m)^{*} T(n)=T\left(m / p^{k-1}\right)^{*} T\left(p^{k-1}\right)^{*} T\left(p^{k}\right) T\left(n / p^{k}\right) \\
= & T\left(m / p^{k-1}\right)^{*} T(p) T\left(n / p^{k}\right)=T(p) T\left(m / p^{k-1}\right)^{*} T\left(n / p^{k}\right)
\end{aligned}
$$

by double commutativity (2) showing that the range of $T(m)^{*} T(n)$ is contained in the range of $T(p)$. Since the range of $T(p)$ is orthogonal to the wandering subspace $\mathcal{E}$ onto which the operator $P$ projects, we conclude that $P T(m)^{*} T(n)=0$ if $n$ does not divide $m$.

REMARK 2.1. Note that

$$
\left.P T(m)^{*} T(n)\right|_{\mathcal{E}}= \begin{cases}I_{\mathcal{E}} & \text { for } n=m, \\ 0 & \text { otherwise. }\end{cases}
$$

Indeed, if $n$ does not divide $m$ we have $P T(m)^{*} T(n)=0$ by Lemma 2.2. If $m$ does not divide $n$ we have by symmetry that

$$
\left.P T(m)^{*} T(n)\right|_{\mathcal{E}}=\left(\left.P T(n)^{*} T(m)\right|_{\mathcal{E}}\right)^{*}=0
$$

by the case already treated.

We associate to an element $x \in \mathcal{H}$ the $\mathcal{E}$-valued Dirichlet series $V x$ defined by the formula

$$
(V x)(s)=\sum_{n=1}^{\infty}\left(P T(n)^{*} x\right) n^{-s},
$$

where $P$ is the orthogonal projection of $\mathcal{H}$ onto the wandering subspace $\mathcal{E}$ for $T$. It is straightforward to see that the series $(V x)(s)$ is absolutely and uniformly convergent in every half-plane $\mathbb{C}_{\alpha}$, where $\alpha>1$. It will in fact follow from our main result Theorem 2.2 below that the series $(V x)(s)$ converges absolutely and uniformly in every half-plane $\mathbb{C}_{\alpha}$, where $\alpha>1 / 2$, provided $T$ satisfies our set of conditions (1)-(3).

We next show that the map $V$ intertwines $T$ with the shift. 
Lemma 2.3. Let $T: \mathbb{Z}^{+} \rightarrow \mathcal{L}(\mathcal{H})$ be a multiplicative semigroup satisfying conditions (1)-(2) and let $V$ be given by (2.5). Then

$$
V T(n) x(s)=n^{-s} V x(s), \quad s \in \mathbb{C}_{1},
$$

for every $x \in \mathcal{H}$ and $n \in \mathbb{Z}^{+}$.

Proof. Notice first that

$$
V T(n) x(s)=\sum_{m \geqq 1}\left(P T(m)^{*} T(n) x\right) n^{-s}, \quad s \in \mathbb{C}_{1} .
$$

By Lemma 2.2

$$
V T(n) x(s)=\sum_{m: n \mid m}\left(P T(m / n)^{*} x\right) m^{-s}=n^{-s} V x(s) .
$$

As mentioned above it will be evident by Theorem 2.2 below that the conclusion of Lemma 2.3 actually holds for $s \in \mathbb{C}_{1 / 2}$.

We are now ready for our main result concerning the map $V$.

Theorem 2.2. Let $T: \mathbb{Z}^{+} \rightarrow \mathcal{L}(\mathcal{H})$ be a multiplicative semigroup satisfying conditions (1)-(3), and let $\mathcal{E}$ given by (2.1) be the wandering subspace for $T$. Then

$$
\|x\|^{2}=\|V x\|_{\mathcal{H}^{2}}^{2}, \quad x \in \mathcal{H},
$$

where $V x$ is given by (2.5). Furthermore, the map $V: x \mapsto V x$ is an isometry mapping $\mathcal{H}$ onto $\mathcal{H}^{2}(\mathcal{E})$ and intertwines $T$ with the shift $S$ in the sense that

$$
V T(n)=S(n) V, \quad n \in \mathbb{Z}^{+} .
$$

Proof. By Lemma 2.3 we know that $V$ intertwines $T$ with $S$. Let us consider the norm identity. Consider an element $x \in \mathcal{H}$ which is a finite sum of the form

$$
x=\sum_{m \geqq 1} T(m) x_{m},
$$

where $x_{m} \in \mathcal{E}$ for $m \geqq 1$. For this element $x$ we have that

$$
P T(n)^{*} x=\sum_{m \geqq 1} P T(n)^{*} T(m) x_{m}=x_{n}
$$

by Remark 2.1. We now have $V x(s)=\sum_{n \geqq 1} x_{n} n^{-s}$, and a calculation of norm gives that

$$
\|V x\|_{\mathcal{H}^{2}}^{2}=\sum_{n \geqq 1}\left\|x_{n}\right\|^{2}
$$


To calculate $\|x\|^{2}$ we again use Remark 2.1 to conclude that

$$
\|x\|^{2}=\sum_{n, m \geqq 1}\left\langle T(m) x_{m}, T(n) x_{n}\right\rangle=\sum_{n, m \geqq 1}\left\langle T(n)^{*} T(m) x_{m}, x_{n}\right\rangle=\sum_{n \geqq 1}\left\|x_{n}\right\|^{2} .
$$

We have now shown that $\|x\|^{2}=\|V x\|^{2}$ for every element $x \in \mathcal{H}$ which is a finite sum of the form (2.6). By Theorem 2.1 the elements $x \in \mathcal{H}$ that are finite sums of the form (2.6) form a dense subset of $\mathcal{H}$. This gives that the map $V$ is an isometry from $\mathcal{H}$ into $\mathcal{H}^{2}(\mathcal{E})$. Notice also that the set of $\mathcal{E}$ valued Dirichlet polynomials form a dense subset of $\mathcal{H}^{2}(\mathcal{E})$. This gives that $V$ maps $\mathcal{H}$ onto $\mathcal{H}^{2}(\mathcal{E})$.

REMARK 2.2. Let $T_{j}: \mathbb{Z}^{+} \rightarrow \mathcal{L}\left(\mathcal{H}_{j}\right)$ be multiplicative semigroups satisfying conditions (1)-(3) for $j=1,2$. It follows by Theorem 2.2 that the semigroups $T_{1}$ and $T_{2}$ are unitarily equivalent in the sense that there exists a unitary operator $U: \mathcal{H}_{1} \rightarrow \mathcal{H}_{2}$ such that

$$
U T_{1}(n)=T_{2}(n) U, \quad n \in \mathbb{Z}^{+},
$$

if and only if the wandering subspaces of $T_{1}$ and $T_{2}$ have the same dimension.

\section{Reproducing kernel functions}

We shall give in this section a second proof of Theorem 2.2 modeled on the use of reproducing kernel functions. First we need a lemma.

Lemma 3.1. Let $T: \mathbb{Z}^{+} \rightarrow \mathcal{L}(\mathcal{H})$ be a multiplicative semigroup satisfying conditions (1)-(3). Then the map $V: \mathcal{H} \ni x \mapsto V x$ defined by (2.5) is injective.

Proof. Vanishing of the function $V x$ means that the element $x \in \mathcal{H}$ is such that $P T(n)^{*} x=0$ for all $n \geqq 1$. Notice that $P T(n)^{*} x=0$ is equivalent to $x \perp T(n) \mathcal{E}$ in $\mathcal{H}$. The result now follows by Theorem 2.1 .

Let us recall the notion of reproducing kernel function. Let $\mathcal{H}$ be a Hilbert space of $\mathcal{E}$-valued functions on a set $\Omega$ such that the point evaluations $\mathcal{H} \ni f \mapsto f(x) \in \mathcal{E}$ at points $x \in \Omega$ are continuous linear operators from $\mathcal{H}$ into $\mathcal{E}$. The reproducing kernel function for the space $\mathcal{H}$ is the function $K_{\mathcal{H}}: \Omega \times \Omega \rightarrow \mathcal{L}(\mathcal{E})$ defined by the properties that $K_{\mathcal{H}}\left(\cdot, x^{\prime}\right) e \in \mathcal{H}$ and

$$
\left\langle f\left(x^{\prime}\right), e\right\rangle=\left\langle f, K_{\mathcal{H}}\left(\cdot, x^{\prime}\right) e\right\rangle, \quad f \in \mathcal{H},
$$

for every $x^{\prime} \in \Omega$ and $e \in \mathcal{E}$, where dot - indicates the variable. 
The reproducing kernel function for the space $\mathcal{H}^{2}(\mathcal{E})$ is given by

$$
K_{\mathcal{H}^{2}(\mathcal{E})}\left(s, s^{\prime}\right)=\zeta\left(s+\bar{s}^{\prime}\right) I_{\mathcal{E}}, \quad s, s^{\prime} \in \mathbb{C}_{1 / 2},
$$

where $I_{\mathcal{E}}$ is the identity operator on $\mathcal{E}$ and

$$
\zeta(s)=\sum_{n=1}^{\infty} n^{-s}
$$

is the Riemann zeta function. Indeed, for $e \in \mathcal{E}, s^{\prime} \in \mathbb{C}_{1 / 2}$ and $f$ given by (0.1) we have

$$
\left\langle f\left(s^{\prime}\right), e\right\rangle=\sum_{n \geqq 1}\left\langle a_{n}, e\right\rangle n^{-s^{\prime}}=\sum_{n \geqq 1}\left\langle f, e n^{-\cdot}\right\rangle n^{-s^{\prime}}=\left\langle f, \zeta\left(\cdot+\overline{s^{\prime}}\right) e\right\rangle,
$$

showing that $K_{\mathcal{H}^{2}(\mathcal{E})}\left(s, s^{\prime}\right) e=\zeta\left(s+\bar{s}^{\prime}\right) e$.

Second Proof of Theorem 2.2. Using the map $V$ we can identify the range $V(\mathcal{H})$ as a Hilbert space of $\mathcal{E}$-valued analytic functions in the half-plane $\mathbb{C}_{1}$. The norm of the function $f=V x$ in $V(\mathcal{H})$ is defined by $\|f\|^{2}=\|x\|^{2}$. Notice that this is well-defined by Lemma 3.1. Notice also that the map $V$ this way becomes an isometry mapping $\mathcal{H}$ onto the space $V(\mathcal{H})$.

We shall compute the kernel function $K_{V(\mathcal{H})}$ for the space $V(\mathcal{H})$ obtained in this way. Let $f=V x$. For $s^{\prime} \in \mathbb{C}_{1}$ and $e \in \mathcal{E}$ we have that

$$
\begin{gathered}
\left\langle f\left(s^{\prime}\right), e\right\rangle=\left\langle V x\left(s^{\prime}\right), e\right\rangle=\sum_{n \geqq 1} n^{-s^{\prime}}\left\langle P T(n)^{*} x, e\right\rangle=\left\langle x, \sum_{n \geqq 1} n^{-\overline{s^{\prime}}} T(n) e\right\rangle \\
=\left\langle V x, V\left(\sum_{n \geqq 1} n^{-\overline{s^{\prime}}} T(n) e\right)\right\rangle=\left\langle f, V\left(\sum_{n \geqq 1} n^{-\overline{s^{\prime}}} T(n) e\right)\right\rangle .
\end{gathered}
$$

By this calculation,

$$
K_{V(\mathcal{H})}\left(s, s^{\prime}\right) e=V\left(\sum_{n \geqq 1} n^{-\overline{s^{\prime}}} T(n) e\right)(s)=\sum_{n, m \geqq 1} m^{-s} n^{-\overline{s^{\prime}}} P T(m)^{*} T(n) e
$$

for $s, s^{\prime} \in \mathbb{C}_{1}$. We now use Remark 2.1 of Lemma 2.2 to conclude that

$$
K_{V(\mathcal{H})}\left(s, s^{\prime}\right) e=\sum_{n \geqq 1} n^{-s-\bar{s}^{\prime}} e .
$$

This shows that $K_{V(\mathcal{H})}\left(s, s^{\prime}\right)=\zeta\left(s+\bar{s}^{\prime}\right) I_{\mathcal{E}}$ for $s, s^{\prime} \in \mathbb{C}_{1}$. It is well-known that the kernel function determines the function space completely (see Aronszajn [3, Section I.2]). We conclude that $V(\mathcal{H})=\mathcal{H}^{2}(\mathcal{E})$ with equality of norms. 
Let $T: \mathbb{Z}^{+} \rightarrow \mathcal{L}(\mathcal{H})$ be a multiplicative semigroup satisfying conditions (1)-(3). For $N \in \mathbb{Z}^{+}$and $x \in \mathcal{H}$ let us consider the function

$$
V_{N} x(s)=P\left(\prod_{k=1}^{N}\left(I-p_{k}^{-s} T\left(p_{k}\right)^{*}\right)^{-1}\right) x, \quad s \in \mathbb{C}_{0},
$$

where $\left\{p_{k}\right\}_{k=1}^{\infty}$ is the sequence of prime numbers and $P$ is the orthogonal projection of $\mathcal{H}$ onto the wandering subspace $\mathcal{E}$ for $T$. Expanding in a geometric series we have

$$
\left(V_{N} x\right)(s)=\sum_{k_{1}, \ldots, k_{N} \geqq 0} P T\left(p_{1}^{k_{1}} \ldots p_{N}^{k_{N}}\right)^{*} x p_{1}^{-k_{1} s} \ldots p_{N}^{-k_{N} s}, \quad s \in \mathbb{C}_{0},
$$

showing that $V_{N} x$ is an $\mathcal{E}$-valued Dirichlet series. It is also evident that $V_{N} x$ belongs to $\mathcal{H}^{2}(\mathcal{E})$ for every $x \in \mathcal{H}$. By Theorem 2.2 we have that

$$
V x=\lim _{N \rightarrow \infty} V_{N} x \quad \text { in } \quad \mathcal{H}^{2}(\mathcal{E})
$$

for every fixed $x \in \mathcal{H}$.

\section{An operator sequence with null wandering subspace}

In this section we shall construct a multiplicative semigroup $T: \mathbb{Z}^{+}$ $\rightarrow \mathcal{L}(\mathcal{H})$ of operators satisfying conditions (1)-(2) but not condition (3) in Section 2. The construction at hand is given by the following theorem.

TheOREM 4.1. Let $\mathcal{H}$ be an infinite dimensional separable Hilbert space and let $\left\{\mathcal{E}_{n}\right\}_{n=0}^{\infty}$ be a sequence of closed subspaces of $\mathcal{H}$ such that $\mathcal{E}_{0}=\mathcal{H}$,

$$
\mathcal{E}_{k} \supset \mathcal{E}_{k+1} \quad \text { and } \quad \operatorname{dim}\left(\mathcal{E}_{k} \ominus \mathcal{E}_{k+1}\right)=+\infty
$$

for every $k \geqq 0$. Then there exists a sequence $\left\{T_{k}\right\}_{k=1}^{\infty}$ of operators in $\mathcal{L}(\mathcal{H})$ such that every operator $T_{k}$ is a pure isometry, the operators $T_{j}$ and $T_{k}$ doubly commute for $j \neq k$, and

$$
\mathcal{E}_{n}=\mathcal{H} \ominus\left(\bigvee_{k=1}^{n} T_{k}(\mathcal{H})\right)
$$

for every $n \geqq 1$. 
Proof. Let us first construct the operator $T_{1}$. As an infinite dimensional separable Hilbert space $\mathcal{H}$ is isomorphic to the vector-valued Hardy space $H^{2}\left(\mathcal{E}_{1}\right)$ and we can further arrange that this isomorphism leaves the subspace $\mathcal{E}_{1}$ fixed. By means of such an isomorphism we transfer the shift on $H^{2}\left(\mathcal{E}_{1}\right)$ to an operator on $\mathcal{H}$. In this way we obtain a pure isometry $T_{1} \in \mathcal{L}(\mathcal{H})$ such that

$$
\mathcal{H}=\bigoplus_{k_{1} \geqq 0} T_{1}^{k_{1}}\left(\mathcal{E}_{1}\right), \quad \mathcal{E}_{1}=\mathcal{H} \ominus T_{1}(\mathcal{H})
$$

where the symbol $\oplus$ denotes orthogonal sum.

Assume next that we have pure isometries $T_{1}, \ldots, T_{n}$ in $\mathcal{L}(\mathcal{H})$ such that $T_{j}$ and $T_{k}$ doubly commute for $j \neq k$ and

$$
\mathcal{H}=\bigoplus_{k_{1}, \ldots, k_{n} \geqq 0} T_{1}^{k_{1}} \ldots T_{n}^{k_{n}}\left(\mathcal{E}_{n}\right), \quad \mathcal{E}_{n}=\mathcal{H} \ominus\left(\bigvee_{k=1}^{n} T_{k}(\mathcal{H})\right)
$$

As an infinite dimensional separable Hilbert space $\mathcal{E}_{n}$ is isomorphic to the vector-valued Hardy space $H^{2}\left(\mathcal{E}_{n+1}\right)$ and we can further assume that this isomorphism leaves the subspace $\mathcal{E}_{n+1}$ fixed. By means of such an isomorphism we transfer the shift on $H^{2}\left(\mathcal{E}_{n+1}\right)$ to an operator on $\mathcal{E}_{n}$. In this way we obtain a pure isometry $T_{n+1} \in \mathcal{L}\left(\mathcal{E}_{n}\right)$ such that

$$
\mathcal{E}_{n}=\bigoplus_{k_{n+1} \geqq 0} T_{n+1}^{k_{n+1}}\left(\mathcal{E}_{n+1}\right), \quad \mathcal{E}_{n+1}=\mathcal{E}_{n} \ominus T_{n+1}\left(\mathcal{E}_{n}\right) .
$$

We next extend this operator $T_{n+1}$ to an operator on $\mathcal{H}$ using the orthogonal sum decomposition (4.1) by requiring that the operators $T_{n+1}$ and $T_{k}$ commute for $k=1, \ldots, n$.

The extended operator $T_{n+1} \in \mathcal{L}(\mathcal{H})$ is clearly a pure isometry since it is a direct sum of pure isometries by the orthogonal sum decomposition (4.1) acting in each component $T_{1}^{k_{1}} \ldots T_{n}^{k_{n}}\left(\mathcal{E}_{n}\right)$ separately.

We shall next prove that $T_{n+1}^{*}$ commutes with $T_{k}$ for $k=1, \ldots, n$. It is apparent that the operator $T_{n+1}$ maps every subspace $T_{1}^{k_{1}} \ldots T_{n}^{k_{n}}\left(\mathcal{E}_{n}\right)$ into itself. By the orthogonal sum decomposition (4.1) this gives that every subspace $T_{1}^{k_{1}} \ldots T_{n}^{k_{n}}\left(\mathcal{E}_{n}\right)$ is reducing for $T_{n+1}$. Write $T^{k^{\prime}}=T_{1}^{k_{1}} \ldots T_{n}^{k_{n}}$. We shall next show that $T_{n+1}^{*} T^{k^{\prime}} e_{n}=T^{k^{\prime}} T_{n+1}^{*} e_{n}$ for $e_{n} \in \mathcal{E}_{n}$. For $e_{n}^{\prime} \in \mathcal{E}_{n}$ we have

$$
\begin{gathered}
\left\langle T_{n+1}^{*} T^{k^{\prime}} e_{n}, T^{k^{\prime}} e_{n}^{\prime}\right\rangle=\left\langle T^{k^{\prime}} e_{n}, T_{n+1} T^{k^{\prime}} e_{n}^{\prime}\right\rangle=\left\langle T^{k^{\prime}} e_{n}, T^{k^{\prime}} T_{n+1} e_{n}^{\prime}\right\rangle \\
=\left\langle e_{n}, T_{n+1} e_{n}^{\prime}\right\rangle=\left\langle T_{n+1}^{*} e_{n}, e_{n}^{\prime}\right\rangle=\left\langle T^{k^{\prime}} T_{n+1}^{*} e_{n}, T^{k^{\prime}} e_{n}^{\prime}\right\rangle,
\end{gathered}
$$


showing that $T_{n+1}^{*} T^{k^{\prime}} e_{n}=T^{k^{\prime}} T_{n+1}^{*} e_{n}$ for $e_{n} \in \mathcal{E}_{n}$ as claimed. By the orthogonal sum decomposition (4.1) it is now evident that $T_{n+1}^{*}$ commutes with $T_{k}$ for $k=1, \ldots, n$.

Let us next prove that $\mathcal{E}_{n+1}=\mathcal{H} \ominus\left(\bigvee_{k=1}^{n+1} T_{k}(\mathcal{H})\right)$. Since the subspace $\mathcal{E}_{n}$ is reducing for $T_{n+1}$ we have that

$$
\mathcal{E}_{n} \ominus T_{n+1}\left(\mathcal{E}_{n}\right)=\mathcal{E}_{n} \ominus T_{n+1}(\mathcal{H})
$$

By (4.1) and (4.2) this gives that

$$
\mathcal{E}_{n+1}=\mathcal{H} \ominus\left(\bigvee_{k=1}^{n+1} T_{k}(\mathcal{H})\right)
$$

Also by (4.1) and (4.2)

$$
\mathcal{H}=\bigoplus_{k_{1}, \ldots, k_{n+1} \geqq 0} T_{1}^{k_{1}} \ldots T_{n+1}^{k_{n+1}}\left(\mathcal{E}_{n+1}\right)
$$

Applying the construction in Theorem 4.1 to a particular sequence of subspaces $\left\{\mathcal{E}_{k}\right\}_{k=0}^{\infty}$ as above such that in addition $\bigcap_{k \geqq 0} \mathcal{E}_{k}=\{0\}$, we obtain a sequence $\left\{T_{k}\right\}_{k=1}^{\infty}$ of pure isometries such that $T_{j}$ and $T_{k}$ doubly commute for $j \neq k$ and

$$
\mathcal{H}=\bigvee_{k=1}^{\infty} T_{k}(\mathcal{H})
$$

Now define $T(n)=T_{1}^{\nu_{1}} \ldots T_{m}^{\nu_{m}}$ where $n=p_{1}^{\nu_{1}} \ldots p_{m}^{\nu_{m}}$ is the factorization of $n \in \mathbb{Z}^{+}$into a product of prime powers. This provides us with a multiplicative semigroup $T: \mathbb{Z}^{+} \rightarrow \mathcal{L}(\mathcal{H})$ satisfying conditions (1)-(2) in Section 2 having null wandering subspace in the sense of (2.1). By Theorem 2.1 this semigroup $T$ does not satisfy condition (3) in Section 2.

\section{Shift invariant subspaces of $\mathcal{H}^{2}$}

As an application of the previous developments we shall describe in this section the shift invariant subspaces $\mathcal{I}$ of $\mathcal{H}^{2}$ having the additional property that

$$
\left.S(p) P_{\mathcal{I}} S(q)^{*}\right|_{\mathcal{I}}=\left.P_{\mathcal{I}} S(q)^{*} S(p)\right|_{\mathcal{I}} \quad \text { in } \quad \mathcal{L}(\mathcal{I})
$$


for all primes $p$ and $q$ with $p \neq q$, where $P_{\mathcal{I}}$ is the orthogonal projection of $\mathcal{H}^{2}$ onto $\mathcal{I}$. Notice that (5.1) says that

$$
\left.S(p)\right|_{\mathcal{I}}\left(\left.S(q)\right|_{\mathcal{I}}\right)^{*}=\left.\left(\left.S(q)\right|_{\mathcal{I}}\right)^{*} S(p)\right|_{\mathcal{I}} \quad \text { in } \quad \mathcal{L}(\mathcal{I})
$$

The description of invariant subspaces we propose uses an appropriate notion of $\mathcal{H}^{2}$-inner function and is analogous to a well-known result for the Hardy space of the unit disc. To avoid technicalities we specialize to the scalar case, that is, $\mathcal{H}^{2}=\mathcal{H}^{2}(\mathbb{C})$ in the notation of the introduction.

Let $\mathcal{I}$ be a shift invariant subspace of $\mathcal{H}^{2}$ and consider the associated semigroup $T: \mathbb{Z}^{+} \rightarrow \mathcal{L}(\mathcal{I})$ defined by

$$
T(n)=\left.S(n)\right|_{\mathcal{I}}, \quad n \in \mathbb{Z}^{+} .
$$

Notice that the assumption (5.1) in essence means that condition (2) of Section 2 holds and that in this context conditions (1) and (3) of Section 2 are automatically satisfied. Notice also that by Theorem 2.2 a shift invariant subspace $\mathcal{I}$ of $\mathcal{H}^{2}$ satisfies (5.1) if and only if the associated semigroup $T$ : $\mathbb{Z}^{+} \rightarrow \mathcal{L}(\mathcal{I})$ given by (5.2) is unitarily equivalent to the shift semigroup $S$ on a space $\mathcal{H}^{2}(\mathcal{E})$ allowing for a general multiplicity $\mathcal{E}$.

Recall the notion of wandering subspace from Section 2. A function $\varphi$ in $\mathcal{H}^{2}$ belongs to a wandering subspace of $\mathcal{H}^{2}$ if and only if it has the orthogonality property that

$$
S(n) \varphi \perp S(m) \varphi \quad \text { in } \quad \mathcal{H}^{2}
$$

for all integers $n, m \in \mathbb{Z}^{+}$with $n \neq m$. By an $\mathcal{H}^{2}$-inner function we mean a function $\varphi$ in $\mathcal{H}^{2}$ of unit norm satisfying (5.3) for all integers $n, m \in \mathbb{Z}^{+}$ with $n \neq m$. It is clearly enough to check (5.3) for relatively prime integers $n, m \in \mathbb{Z}^{+}$with $n \neq m$.

Functions in $\mathcal{H}^{2}$ have a natural description using Fourier expansions of $L^{2}$-functions living on the character group $\Xi$. By a character we mean a function $\chi: \mathbb{Z}^{+} \rightarrow \mathbb{T}$ such that

$$
\chi(n m)=\chi(n) \chi(m), \quad n, m \in \mathbb{Z}^{+},
$$

and we denote by $\Xi$ the set of all such characters $\chi$. By defining $\chi(r)$ $=\chi(n) / \chi(m)$ for $r=n / m \in \mathbb{Q}^{+}$it is straightforward to see that characters $\chi \in \Xi$ correspond to group homomorphisms $\chi: \mathbb{Q}^{+} \rightarrow \mathbb{T}$ from the multiplicative group of positive rational numbers $\mathbb{Q}^{+}$to the unit circle $\mathbb{T}$. We equip $\mathbb{Q}^{+}$ with the discrete topology in order to make its dual group $\Xi$ compact. The 
character group $\Xi$ is easily seen to be isomorphic to the infinite dimensional torus $\mathbb{T}^{\omega}$ by means of the map

$$
\Xi \ni \chi \mapsto\left\{\chi\left(p_{k}\right)\right\}_{k=1}^{\infty} \in \mathbb{T}^{\omega}
$$

where $\left\{p_{k}\right\}_{k=1}^{\infty}$ is the sequence of prime numbers. We mention also that the character group $\Xi$ is universal in the sense that it contains every compact metrizable abelian group as a subgroup (see [19, Theorem 2.2.6]).

The Haar measure on $\Xi$ is denoted by $\mathrm{d} \chi$ and is normalized to have unit mass. We write $e_{r}\left(r \in \mathbb{Q}^{+}\right)$for the functions

$$
e_{r}(\chi)=\chi(r), \quad \chi \in \Xi
$$

which are known as the exponential monomials on $\Xi$. In this context the Plancherel formula takes the form

$$
\int_{\Xi}|f(\chi)|^{2} d \chi=\sum_{r \in \mathbb{Q}^{+}}|\hat{f}(r)|^{2}, \quad f \in L^{2}(\Xi),
$$

where

$$
\hat{f}(r)=\int_{\Xi} f(\chi) \overline{e_{r}(\chi)} d \chi, \quad r \in \mathbb{Q}^{+},
$$

are the Fourier coefficients of a, say, integrable function $f$ on $\Xi$. For background information on Fourier analysis on locally compact abelian groups we refer to the classical book by Rudin [19].

By means of Fourier transformation the map $f \mapsto \mathcal{D} f$, where

$$
\mathcal{D} f(s)=\sum_{n=1}^{\infty} \hat{f}(n) n^{-s}, \quad s \in \mathbb{C}_{1 / 2},
$$

gives an isometric isomorphism of the subspace

$$
H^{2}(\Xi)=\left\{f \in L^{2}(\Xi): \hat{f}(r)=0 \text { for } r \in \mathbb{Q}^{+} \backslash \mathbb{Z}^{+}\right\}
$$

of $L^{2}(\Xi)$ onto $\mathcal{H}^{2}$. Notice that $\mathcal{D}$ intertwines the operator on $H^{2}(\Xi)$ given by multiplication by the exponential monomial $e_{n}$ with the shift $S(n)$ acting on $\mathcal{H}^{2}$. When clear from the context we suppress the operator $\mathcal{D}$ and think of $f \in H^{2}(\Xi)$ as a boundary value of $\mathcal{D} f \in \mathcal{H}^{2}$ as is customary in complex analysis.

Proposition 5.1. A function $\varphi$ in $\mathcal{H}^{2}$ is $\mathcal{H}^{2}$-inner if and only if $|\varphi(\chi)|=1$ for a.e. $\chi \in \Xi$. 
Proof. We reproduce a classical argument. For integers $n, m \in \mathbb{Z}^{+}$we have that

$$
\langle S(n) \varphi, S(m) \varphi\rangle=\int_{\Xi} e_{n}(\chi) \varphi(\chi) \overline{e_{m}(\chi) \varphi(\chi)} d \chi=\int_{\Xi}|\varphi(\chi)|^{2} \overline{e_{m / n}(\chi)} d \chi .
$$

By this $\varphi$ is $\mathcal{H}^{2}$-inner if and only if $\left(|\varphi|^{2}-1\right)^{\wedge}(r)=0$ for all $r \in \mathbb{Q}^{+}$, which is equivalent to $|\varphi(\chi)|=1$ for a.e. $\chi \in \Xi$.

We next describe the wandering subspaces of $\mathcal{H}^{2}$.

Lemma 5.1. Every nonzero wandering subspace $\mathcal{E}$ of $\mathcal{H}^{2}$ is one dimensional and is spanned by an $\mathcal{H}^{2}$-inner function $\varphi$.

Proof. It is evident that every function $\varphi$ in $\mathcal{E}$ of unit norm is $\mathcal{H}^{2}$-inner. Let $\varphi_{1}, \ldots, \varphi_{n}$ be an orthonormal set of functions from $\mathcal{E}$. We shall prove that for a.e. $\chi \in \Xi$ the map

$$
\Lambda: \mathbb{C}^{n} \ni\left(a_{1}, \ldots, a_{n}\right) \mapsto \sum_{j=1}^{n} a_{j} \varphi_{j}(\chi) \in \mathbb{C}
$$

is an isometry of $\mathbb{C}^{n}$ onto $\mathbb{C}$. By elementary linear algebra this gives the conclusion of the lemma.

Let $\left(a_{1}, \ldots, a_{n}\right) \in \mathbb{C}^{n}$ be of unit norm. Then the function $\varphi=\sum_{j=1}^{n} a_{j} \varphi_{j}$ belongs to $\mathcal{E}$ and is of unit norm and so is $\mathcal{H}^{2}$-inner. By Proposition 5.1 we have that $|\varphi(\chi)|=1$ for a.e. $\chi \in \Xi$. This proves that the map $\Lambda$ is an isometry for a.e. $\chi \in \Xi$.

We next describe the shift invariant subspaces $\mathcal{I}$ of $\mathcal{H}^{2}$ satisfying (5.1).

THEOREM 5.1. Let $\mathcal{I}$ be a nonzero shift invariant subspace of $\mathcal{H}^{2}$. Then $\mathcal{I}$ satisfies (5.1) for all primes $p$ and $q$ with $p \neq q$ if and only if it has the form

$$
\mathcal{I}=\varphi \mathcal{H}^{2}=\left\{\varphi g: g \in \mathcal{H}^{2}\right\}
$$

for some $\mathcal{H}^{2}$-inner function $\varphi$. The $\mathcal{H}^{2}$-inner function $\varphi$ in (5.4) is uniquely determined up to a unimodular constant.

Proof. If $\mathcal{I}$ has the form $\mathcal{I}=\varphi \mathcal{H}^{2}$ for some $\mathcal{H}^{2}$-inner function $\varphi$, then multiplication by $\varphi$ provides a unitary equivalence of $\mathcal{H}^{2}$ onto $\mathcal{I}$. By Proposition 1.1 this gives that (5.1) holds.

Suppose next that $\mathcal{I}$ is a shift invariant subspace of $\mathcal{H}^{2}$ satisfying (5.1). It is evident that the associated semigroup $T$ given by (5.2) satisfies conditions (1)-(3) of Section 2. Also denote by

$$
\mathcal{E}=\mathcal{I} \ominus\left(\bigvee_{k=1}^{\infty} T\left(p_{k}\right)(\mathcal{I})\right)
$$


the wandering subspace for $\mathcal{I}$. By Theorem 2.2 a function $f$ belongs to $\mathcal{I}$ if and only if it has the form

$$
f(s)=\sum_{n=1}^{\infty} \varphi_{n}(s) n^{-s}, \quad s \in \mathbb{C}_{1 / 2},
$$

for some sequence $\left\{\varphi_{n}\right\}_{n=1}^{\infty}$ of functions from $\mathcal{E}$ such that $\sum_{n=1}^{\infty}\left\|\varphi_{n}\right\|^{2}<+\infty$, and furthermore the norm identity

$$
\|f\|^{2}=\sum_{n=1}^{\infty}\left\|\varphi_{n}\right\|^{2}
$$

holds. By Lemma 5.1, $\varphi_{n}=a_{n} \varphi$ for some constants $a_{n} \in \mathbb{C}$, where $\varphi$ is an $\mathcal{H}^{2}$-inner function from $\mathcal{E}$. Now

$$
f(s)=\sum_{n=1}^{\infty} a_{n} \varphi(s) n^{-s}=\varphi(s) g(s), \quad s \in \mathbb{C}_{1 / 2},
$$

where $g(s)=\sum_{n=1}^{\infty} a_{n} n^{-s}, s \in \mathbb{C}_{1 / 2}$, is a function in $\mathcal{H}^{2}$ with $\|g\|^{2}=\|f\|^{2}$. This gives the description (5.4).

If $\mathcal{I}$ has the form (5.4) for some $\mathcal{H}^{2}$-inner function $\varphi$, then a straightforward calculation shows that $\varphi$ belongs to the wandering subspace $\mathcal{E}$ for $\mathcal{I}$ given by (5.5) above. The last uniqueness assertion now follows by Lemma 5.1.

We remark that a polydisc version of Theorem 5.1 is due to Agrawal, Clark and Douglas [1]; see also Mandrekar [14].

An important feature of $\mathcal{H}^{2}$-inner functions is that such functions are the isometric multipliers on $\mathcal{H}^{2}$. It should be mentioned here a result by Hedenmalm, Lindqvist and Seip [11, Theorem 3.1] which characterizes the bounded multipliers $m$ on $\mathcal{H}^{2}$ as Dirichlet series

$$
m(s)=\sum_{n=1}^{\infty} m_{n} n^{-s}, \quad s \in \mathbb{C}_{1 / 2},
$$

that extend analytically to bounded analytic functions in the right halfplane $\mathbb{C}_{0}$. The operator norm of such a multiplier $m$ equals its supremum

$$
\|m\|=\sup _{s \in \mathbb{C}_{0}}|m(s)|
$$

in $\mathbb{C}_{0}$, and the space of such Dirichlet series $m$ is denoted $\mathcal{H}^{\infty}$. See also [12] for some historical comments. In this relation we feel that the subclass of $\mathcal{H}^{\infty}$ consisting of $\mathcal{H}^{2}$-inner functions deserves further attention. 
To this end we wish to mention that function spaces derived from $\mathcal{H}^{2}$ have attracted quite some interest recently with contributions by Bayart, Hedenmalm, McCarthy, Saksman, Seip, Queffélec and others, see for instance $[4,5,8,10,13,15,17,20]$ in the references.

\section{References}

[1] O. P. Agrawal, D. N. Clark and R. G. Douglas, Invariant subspaces in the polydisk, Pacific J. Math., 121 (1986), 1-11.

[2] A. Aleman, S. Richter and C. Sundberg, Beurling's theorem for the Bergman space, Acta Math., 177 (1996), 275-310.

[3] N. Aronszajn, Theory of reproducing kernels, Trans. Amer. Math. Soc., 68 (1950), 337-404.

[4] F. Bayart, Hardy spaces of Dirichlet series and their composition operators, Monatsh. Math., 136 (2002), 203-236.

[5] F. Bayart, S. V. Konyagin and H. Queffélec, Convergence almost everywhere and divergence everywhere of Taylor and Dirichlet series, Real Anal. Exchange, 29 (2003/04), 557-586.

[6] H. Bohr, Über die Bedeutung der Potenzreihen unendlich vieler Variabeln in der Theorie der Dirichletschen Reihen $\sum a_{n} / n^{s}$, Nachr. Akad. Wiss. Göttingen Math.Phys. Kl. (1913), 441-488.

[7] B. J. Cole and T. W. Gamelin, Representing measures and Hardy spaces for the infinite polydisk algebra, Proc. London Math. Soc. (3), 53 (1986), 112-142.

[8] J. Gordon and H. Hedenmalm, The composition operators on the space of Dirichlet series with square summable coefficients, Michigan Math. J., 46 (1999), 313329 .

[9] P. R. Halmos, Shifts on Hilbert spaces, J. Reine Angew. Math., 208 (1961), 102-112.

[10] H. Hedenmalm, Dirichlet series and functional analysis, in: The legacy of Niels Henrik Abel, Springer (2004), pp. 673-684.

[11] H. Hedenmalm, P. Lindqvist and K. Seip, A Hilbert space of Dirichlet series and systems of dilated functions in $L^{2}(0,1)$, Duke Math. J., 86 (1997), 1-37.

[12] H. Hedenmalm, P. Lindqvist and K. Seip, Addendum to: "A Hilbert space of Dirichlet series and systems of dilated functions in $L^{2}(0,1)$ ", Duke Math. J., 99 (1999), $175-178$.

[13] H. Hedenmalm and E. Saksman, Carleson's convergence theorem for Dirichlet series, Pacific J. Math., 208 (2003), 85-109.

[14] V. Mandrekar, The validity of Beurling theorems in polydiscs, Proc. Amer. Math. Soc., 103 (1988), 145-148.

[15] J. E. McCarthy, Hilbert spaces of Dirichlet series and their multipliers, Trans. Amer. Math. Soc., 356 (2004), 881-893.

[16] A. Olofsson, A von Neumann-Wold decomposition of two-isometries, Acta Sci. Math. (Szeged), 70 (2004), 715-726.

[17] J.-F. Olsen and K. Seip, Local interpolation in Hilbert spaces of Dirichlet series, Proc. Amer. Math. Soc., 136 (2008), 203-212.

[18] D. Popovici, A Wold-type decomposition for commuting isometric pairs, Proc. Amer. Math. Soc., 132 (2004), 2303-2314.

[19] W. Rudin, Fourier Analysis on Groups, Wiley (1962).

[20] E. Saksman and K. Seip, Integral means and boundary limits of Dirichlet series, Bull. London Math. Soc., 41 (2009), 411-422. 
[21] S. Shimorin, Wold-type decompositions and wandering subspaces for operators close to isometries, J. Reine Angew. Math., 531 (2001), 147-189.

[22] M. Słociński, On the Wold-type decomposition of a pair of commuting isometries, Ann. Polon. Math., 37 (1980), 255-262.

[23] B. Sz.-Nagy and C. Foias, Harmonic Analysis of Operators on Hilbert Space, NorthHolland (1970). 